\title{
Effect of Conjugated Linoleic Acid on Testosterone LeVels In Vitro AND In Vivo After an Acute Bout of Resistance Exercise
}

\author{
Filippo Macaluso, ${ }^{1,2}$ Giuseppe Morici, ${ }^{2}$ Patrizia Catanese, ${ }^{2}$ Nella M. Ardizzone, ${ }^{2}$ \\ Antonella Marino Gammazza, ${ }^{2}$ Giuseppe Bonsignore, ${ }^{3}$ Giuseppe Lo Giudice, ${ }^{4}$ \\ Tomaso Stampone, ${ }^{4}$ Rosario Barone, ${ }^{2}$ Felicia Farina, ${ }^{2}$ and Valentina Di Felice ${ }^{2}$ \\ ${ }^{1}$ Department of Physiological Science, Stellenbosch University, Stellenbosch, South Africa; ${ }^{2}$ Department of Experimental \\ Biomedicine and Clinical Neroscience (BioNeC), University of Palermo, Palermo, Italy; ${ }^{3}$ Laboratory of Transfusion Medicine; \\ and ${ }^{4}$ Laboratory of Clinical Pathology, Hospital "Villa Sofia-CTO," Palermo, Italy
}

\begin{abstract}
Macaluso, F, Morici, G, Catanese, P, Ardizzone, NM, Marino Gammazza, A, Bonsignore, G, Giudice, GL, Stampone, T, Barone, R, Farina, F, and Di Felice, V. Effect of conjugated linoleic acid on testosterone levels in vitro and in vivo after an acute bout of resistance exercise. J Strength Cond Res 26(6): 1667-1674, 2012-The purposes of the present study were to investigate the effect of conjugated linoleic acid (CLA) supplementation on testosterone levels in vitro on a cell line derived from Leydig cells (R2C) and in vivo in the blood of physically active subjects before and after a resistance exercise bout. In vitro R2C cells were treated with different CLA concentrations $(0-30 \mu \mathrm{M})$ for 24 and 48 hours. After treatment, supernatant media were tested to determine testosterone secretion. The CLA increased the testosterone secretion only after 48 hours. In vivo, 10 resistance-trained male subjects, in a double-blind placebo-controlled and crossover study design were randomized for 3 weeks of either $6 \mathrm{~g} \cdot \mathrm{d}^{-1}$ CLA or placebo. Blood was drawn pre and post each resistance exercise bout to determine the total testosterone and sex hormone-binding globulin (SHBG) levels. No significant differences were observed for total testosterone or SHBG pre and post each resistance exercise bout; although after the resistance exercise bouts, total testosterone increased moderately (effect size $=$ moderate), whereas after CLA supplementation, there was a large increase in total testosterone (effect size $=$ large). CLA supplementation induced an increase in testosterone levels in Leydig cells in vitro after 48 hours but not in vivo before and after a resistance exercise
\end{abstract}

F. Macaluso and G. Morici contributed equally to the present work. Address correspondence to Filippo Macaluso, filippo.mac@libero.it.

26(6)/1667-1674

Journal of Strength and Conditioning Research

(C) 2012 National Strength and Conditioning Association bout. These findings suggest that CLA supplementation may promote testosterone synthesis through a molecular pathway that should be investigated in the future, although this effect did not have an anabolic relevance in our in vivo model.

KEY WoRDS food supplement, hormones, body composition, Leydig cell

\section{INTRODUCTION}

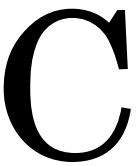
onjugated linoleic acid (CLA) is a group of positional and geometric isomers of linoleic acid (cis-9, cis-12-octadecadienoic acid), an omega-6 essential fatty acid. The characteristic features of these isomers are that, unlike linoleic acid, the 2 double bonds along the 18-carbon fatty acid chain are separated by a single carbon-carbon bond, not a methylene group. The 2 main isomers of CLA are cis- 9 , trans- 11 and trans-10,cis-12 octadecaenoic acids (c9,t11-CLA, t10,c12-CLA) (16).

The use of CLA supplements is spreading among elite and recreational athletes because marketing claims suggest that it can improve endurance capacity, increase $\dot{\mathrm{V}}_{\mathrm{O}_{2}}$ max, reduce body mass, reduce muscle glycogen breakdown, improve metabolism, prevent or reduce muscle damage and the inflammatory response, promote fat loss, and reduce catabolism $(15,18)$. Although only a few studies have been conducted to evaluate the effects of CLA supplementation and exercise training (endurance and resistance) in animal and human subjects. In animals, it has been demonstrated that CLA supplementation combined with endurance training augments the positive effect of training, that is, decreases body weight (11) and adipose tissue $(1,11)$ and increases lean body mass (1), muscle hypertrophy (7), and endurance performance (19). Although the results in animals show that CLA supplementation has positive effects, the studies conducted with humans show contradictory results. Kreider et al. (18) and Pinkoski et al. (21), who investigated the effects of CLA supplementation during resistance exercise training on subjects performing different sporting 
activities, observed different results despite the fact that both performed randomized controlled studies. Conjugated linoleic acid supplementation in experienced resistance-trained athletes did not induce differences in total body mass, body composition, and strength (18), whereas in moderately physically active subjects, CLA induced a relatively small increase in lean body mass and a decrease in body fat mass (21).

The effect of CLA supplementation on body fat mass reduction has been investigated (5), and in in vitro studies, the lipolytic effect of CLA on adipocytes seems to be mediated by an increase in the levels of a protein named perilipin $A(6)$. The proteins of the perilipin family are found not only on the outer surface of lipid storage droplets of adipocytes (linked to the hormone-sensitive lipase) but also in steroidogenic cells, such as Leydig cells in testicles, associated to the cholesteryl esterase (22). The steroidogenic cells' cholesteryl esters, precursors of testosterone, are stored in the intracellular lipid droplets of Leydig cells (22). Moreover, it has previously been suggested that exercise training might alter the amount and the function of perilipin (20).

Considering the lipolytic effect of CLA, the role of perilipins in the pathway leading to steroid hormone synthesis and the effect of exercise on perilipin function, it was hypothesized that CLA supplementation may mediate an increase in testosterone secretion. The purposes of the study were (a) to investigate the effect of CLA supplementation on testosterone synthesis in Leydig cells, an in vitro model for studying steroid biosynthesis, and (b) to test the effect of CLA supplementation on testosterone levels in vivo in the blood of physical active subjects and after a resistance exercise bout. We suppose that the results from this study will lead the practitioners to understand if the CLA supplementation can be useful to increase the testosterone synthesis immediately after an acute session of resistance exercise affecting the anabolic phase followed by each strength training session.

\section{Methods}

\section{Experimental Approach to the Problem}

To test if CLA supplementation has an effect on the synthesis of testosterone, we approached the problem using in vitro and in vivo models.

\section{Subjects}

For the first model, we used a Leydig cell line, which is steroidogenic without cAMP stimulation (22). Investigation of the effect of CLA on a rat cell line was done because (a) it is unethical to perform a testicular biopsy in humans without any prior results from animal studies that justify them; (b) it is impossible to obtain a 100\% pure Leydig cell culture from animal testes, and these cells are hard to maintain in culture, unlike the cell line used; and (c) only the $\mathrm{R} 2 \mathrm{C}$ cell at a basal conditioning level produces steroid hormones, simulating the activation of these cells in humans after a resistance exercise bout, while Leydig cells isolated from animal testis require the addition of human chorionic gonadotropin to produce steroid hormones. The cells were treated with different concentration of CLA, and the supernatant medium was tested after 24 and 48 hours to determine total testosterone synthesis.

The human model was conducted as a crossover, doubleblind, placebo-controlled clinical trial. All subjects gave the investigators informed consent before participating in the study. Subjects were assigned to condition using randomization and provided with CLA or placebo for 3 weeks. After 2 weeks of washout, the groups were switched and all subjects received the opposite substance for an additional 3 weeks. Before and after each supplementation period, the subjects underwent a resistance exercise bout and body composition analysis. Blood samples were collected for hormone analyses before and after each resistance exercise bout.

The concentration of CLA supplementation administered for the in vivo experiment was based on doses previously used in literature and not on the doses used in the in vitro experiment because it is unethical to perform a testicular biopsy to verify actual CLA concentration of the testis. The study was conducted in Sicily (Italy) during springtime.

\section{A: In Vitro}

Cell Cultures. Rat Leydig Cells (R2C, cat. No. 89031606, ECACC; Health Protection Agency Culture Collections, Salisbury, United Kingdom) were cultured in $75-\mathrm{cm}^{2}$ flasks in M-199 medium (Invitrogen Corp., Carlsbad, CA, USA) supplemented with 15\% horse serum (Invitrogen Corp), 2.5\% fetal bovine serum (Invitrogen Corp), and antibiotic antimycotic solution (penicillin $100 \mathrm{U} \cdot \mathrm{ml}^{-1}$, streptomycin $100 \mu \mathrm{g} \cdot \mathrm{ml}^{-1}$, amphotericin B $0.25 \mu \mathrm{g} \cdot \mathrm{ml}^{-1}$; Invitrogen Corp). Cells were incubated at $37^{\circ} \mathrm{C}$ in a humidified atmosphere with $5 \% \mathrm{CO}_{2}$ and fed 3 times a week until reaching 80 to $90 \%$ confluence. Adherent cells were harvested with trypsin in $\mathrm{Ca}^{2+}$ - and $\mathrm{Mg}^{2+}$-free Hanks' balanced salt solution (Invitrogen Corp), washed with phosphate-buffered saline and resuspended in the supplemented M-199 medium.

Conjugated Linoleic Acid Treatment. When cells reached about $80 \%$ confluence, they were treated with the CLA working solution at increasing concentrations $(0-30 \mu \mathrm{M}$, to reduce the error of the operator, we used dilution series 1:2 starting from the $30 \mu \mathrm{M}$ concentration) for 24 and 48 hours. To prepare the CLA working solution, the Tonalin FFA 80 (Cognis Nutrition and Health) was first dissolved in absolute ethanol and then in fetal bovine serum containing 1\% bovine serum albumin (Sigma-Aldrich, St. Louis, MO, USA).

Viability Test. The CLA working solution concentrations used were tested with a 3-(4,5-dimethylthiazol-2-yl)-5-(3-carboxymethoxyphenyl)-2-(4-sulfophenyl)-2H-tetrazolium assay (MTS test). For the MTS assay, the CellTiter 96 Aqueous One Solution Cell Proliferation Assay kit (Promega Corporation, Madison, WI, USA) was used following the manufacturer's instruction. Briefly, cells were seeded at $5 \times 10^{3}$ cells per well in 96-well plates, and after the incubation period of 24 and 48 hours with the CLA working solution, $20 \mu \mathrm{L}$ of the MTS 
Journal of Strength and Conditioning Research $\mid$ www.nsca-jscr.org

reagent was added to each well and cells were incubated at $37^{\circ} \mathrm{C}$ for 3 hours. At the end of the incubation period, the absorbance of the formazan produced was detected at $490 \mathrm{~nm}$ with a multiwell plate reader (DV990BV4; GDV, Rome, Italy). CLA induced cell death at concentrations higher than $15 \mu \mathrm{M}$ after 48 hours (30-40\% of mortality). Hence, to study the synthesis of testosterone in vitro, we used concentrations up to $7.5 \mu \mathrm{M}$. The MTS test was performed in quintuplicate.

Testosterone Analysis. Cells were seeded at $5 \times 10^{4}$ cells per well in 24-well plates. To determine testosterone secretion, the medium was collected at the end of the incubation period and subsequently analyzed with an Immulite 2000 (Medical System S.P.A., Genova, Italy). Total testosterone analysis in cell culture was performed in triplicate in 5 different independent experiments.

\section{B: In Vivo}

Subjects. Ten physically active male subjects (age, $27.4 \pm 3.7$ years; height, $176.7 \pm 5.2 \mathrm{~cm}$; weight, $81.1 \pm 9.8 \mathrm{~kg}$; body mass index (BMI), $25.9 \pm 2.6 \mathrm{~kg} \cdot \mathrm{m}^{-2}$; body fat, $15.1 \pm 3.1 \%$ ) volunteered to participate in this investigation. Subjects were informed of the experimental procedures and associated risk before providing written informed consent. This study conformed to the standards set by the latest revision of the Declaration of Helsinki, and it was approved by the institutional review board for the protection of human subjects of the University of Palermo. Each subject had more than 2 years of resistance training experience and had been training at least 3 times per week over the last year. All subjects completed a health history questionnaire, were healthy, and had no medical contraindications or history of any endocrine disorders. The subjects were not vegetarians, nor did they follow a carbohydrate-restricted diet. No subjects were taking any medications or nutritional supplements, and all were nonsteroid users and nonsmokers. Baseline hormones and sex hormone-binding globulin (SHBG) blood levels are summarized in Table 1.

Experimental Design. This study was conducted as a crossover, double-blind, placebo-controlled, clinical trial. The subjects were randomized to groups receiving either CLA (see below for dosage) or placebo (PLA) for 3 weeks. After 2 weeks of washout, the groups were switched and all subjects received the opposite substance for an additional 3 weeks. Before and after each supplementation period, the subjects underwent body composition analysis and a resistance exercise bout. During the 6 weeks ( 3 weeks $\times 2$ times) of supplementation, the subjects were instructed to consume their regular diet (carbohydrate, $57.5 \pm 5.0 \%$; lipid, $15.0 \pm 2.5 \%$; protein, $25.5 \pm$ $1.5 \%$ ), to maintain their usual training routine (resistance training 3 times per week, $60 \pm 7.5$ minutes per training session), and to maintain normal nocturnal sleep habits ( $8 \pm 1$ hour per night). No differences were observed during the study period in macronutrients ingested, training hours per session, and nocturnal sleep hours per night (analysis performed on diet, training, and sleep questionnaires filled out daily by the subjects). It was recommended that they abstain from vigorous exercise, alcohol use, and sexual activity for at least 24 hours before the resistance exercise bout. All the subjects declared to abstain from these activities 24 hours before the resistance exercise bout.

Conjugated Linoleic Acid and PLA Supplementation. The subjects ingested 7 opaque soft gel capsules of CLA (6 g of Tonalin FFA 80; Cognis Group, Monheim, Germany) or PLA (6 $g$ of sunflower oil) per day, both identical in taste and in appearance. The CLA and placebo dosages were used in literature in studies that investigated the effect of CLA supplementation on body mass composition $(18,21)$; moreover, this CLA dose is suggested by the food supplement manufacturers. The CLA supplement was a mixture containing 40\% c9,t11-CLA and $40 \% \mathrm{t} 10, \mathrm{c} 12-\mathrm{CLA}$. The rest of the mixture was made of other fatty acids $(10 \%$ of oleic acid, $<4 \%$ of palmitic acid, $<4 \%$ of stearic acid, and $<3 \%$ of linoleic acid). At the beginning of each supplementation period, the subjects received 3 boxes with 56 pills each. At the end of each supplementation period, the remaining pills per box were counted to verify the supplement intake. Subjects consumed $99.6 \pm 1.3 \%$ and $99.2 \pm 1.7 \%$ of the prescribed intake during the CLA and PLA supplementation periods, respectively.

1 Repetition Maximum Strength Test. Maximal strength was determined for each of the 8 exercises of the resistance protocol (leg press, leg curl, leg extension, lat pull-down, bench press, shoulder press, barbell bicep curl, and supine triceps extension). This was assessed by completing a 1 repetition maximum (1-RM) strength test (i.e., the heaviest load that can be lifted one time through a full range of motion). Three to 4 subsequent attempts were performed to determine the 1-RM strength, with the weight increasing progressively until the subjects failed at the given load. The 1-RM strength test was preceded by a warm-up 
session consisting of 10 -minute cycling on a stationary bike, 5 -minute light stretching, and 2 to 3 sets of 8 to 10 repetitions of leg press at $<50 \%$ of perceived maximal strength. 1 Repetition maximum strength tests were performed 1 week before the start of each resistance bout protocol.

Resistance Exercise Bout Protocol. The resistance exercise bout consisted of 8 resistance exercises. The subject's goal was to complete 3 sets of 8 to 10 repetitions at approximately $75 \%$ of that subject's 1-RM strength. One minute of rest between each set and 2 minutes between each exercise were allowed for recovery. The number of repetitions and the amount of weight lifted in each exercise were recorded to calculate the total lifting volume, that is, the training volume.

A warm-up was performed before each resistance exercise bout. This resistance exercise protocol was based on Bird et al. (3) and was chosen because (a) it involves a large muscle mass at high intensity simulating training of recreational gymnasium users, differently from other protocols proposed in literature, and (b) it increases (around 30\%) the total testosterone concentration in men until the end of the resistance exercise bout. The resistance exercise bouts and the 1-RM strength test were performed under the supervision of qualified personal trainers.

Body Composition Analysis. Body composition analysis was performed early in the morning after a night of fasting on the first and the last day of each supplementation period with the Inbody 320 (BioSpace, Seoul, Korea), a bioelectrical impedance analyzer. The device uses 8 points of tactile electrodes (contact at the hands and feet) and 3 frequencies $(5,50$, and $200 \mathrm{kHz}$ ) to measure body mass (kilograms), body fat mass (kilograms), lean body mass (kilograms) and intra- and extracellular water (14). All subjects were asked to empty their bladders immediately before the measurements. The bioelectrical impedance analysis was performed with subjects dressed only in their underwear (with all jewelery removed). The validity and reliability of this specific bioelectrical impedance analyzer in adult men has been reported by Jensky-Squires et al. (14).

Blood Sampling and Analysis. Each resistance exercise bout was performed at 4:00 PM after a 4-hour fast, to minimize the influence of diurnal variations on exercise performance and hormonal response (2). Blood was drawn before (pre) and after (post) each resistance exercise bout from the antecubital vein to determine hormone concentrations (total testosterone, estradiol, and cortisol) and SHBG levels. Subjects sat quietly for 15 minutes, after which a butterfly needle was inserted into the antecubital forearm vein and the pre blood samples were drawn. The subjects remained sitting quietly for another 15 minutes, to minimize hormonal fluctuations related to anticipatory responses. The post blood samples were drawn 5 minutes after completion of the resistance exercise bout (2). The tubes were gently inverted 5 times and allowed to stand at room temperature for a minimum of 20 minutes. Samples were then centrifuged for 10 minutes at 3,000 revolutions per minute, with the supernatant removed, placed into plastic storage containers, and frozen at $-80^{\circ} \mathrm{C}$ until analysis. Percentage changes in plasma and red cell volume were calculated using hemoglobin and hematocrit concentrations, according to Dill and Costill (8). Blood measurements were performed by the hematology laboratory of "Azienda Ospedaliera Villa Sofia-CTO Palermo." The hormones and SHBG were measured on an Immulite 2000 (Siemens, Milan, Italy) using the manufacturer's instruction, fully described previously (10). Briefly, the automated instrument dropped a single assay-specific bead into a sample tube that was agitated at $37^{\circ} \mathrm{C}$. After the sample tube was agitated at $37^{\circ} \mathrm{C}$, then washed and a chemiluminescent substrate added. The amount of light generated was measured by the photomultiplier tube, and the quantity of hormone was calculated by the software of the instrument. Inter- and intra-assay coefficients of variances were 9.5 and $8.3 \%$ for total testosterone (Immunolite 2000 testosterone total), 9.0 and $6.2 \%$ for estradiol (Diasorin estradiol, Liaison estradiol, DiaSorin, Stillwater, MN, USA), 7.0 and $5.5 \%$ for cortisol (Immunolite 2000 cortisol), and 4.2 and $3.0 \%$ for SHBG (Immunolite 2000 SHBG).

\section{Statistical Analyses}

One-way analysis of variance (ANOVA) for different CLA concentrations was performed on testosterone secretion by R2C cells. Pearson's correlation was performed for testosterone synthesis in vitro. Body composition measurements and mean training volumes were analyzed by 2-way ANOVA for repeated measures: supplementation (CLA and PLA) vs. training (before and after). Hormones and SHBG measurements were analyzed by 3 -way ANOVA with repeated measures: supplementation vs. training vs. resistance exercise bout (pre and post). Cohen's effect size (ES) was also calculated to determine the effect of supplementation on testosterone level $(\mathrm{ES} \leq 0.2$, small; $0.2<\mathrm{ES} \leq 0.6$, moderate; $\mathrm{ES}>0.6$, large). Assumptions for ANOVA were assessed, and ANOVAs were robust to violation of normality and homogeneity. If a significant difference was detected during 1-, 2- or 3-way ANOVAs, this was further evaluated by post hoc Tukey's test. Confidence intervals (90\%) for baseline values and normal ranges of hormones and SHBG blood level are summarized in Table 1 . Statistical analyses were performed using Statistica (StatSoft, Inc, Tulsa, OK, USA). All data are presented as means $\pm S E M$, and the level of statistical significance was set at $p \leq 0.05$.

\section{Results}

\section{Testosterone Production In Vitro}

In the $\mathrm{R} 2 \mathrm{C}$ cells, the CLA working solution induced an increase in the concentration of the secreted testosterone within 48 hours (Figure 1). Testosterone secretion increased linearly with CLA supplementation $\left(R^{2}=0.520, p<0.018\right)$. This effect was not evident at 24-hour treatment. 


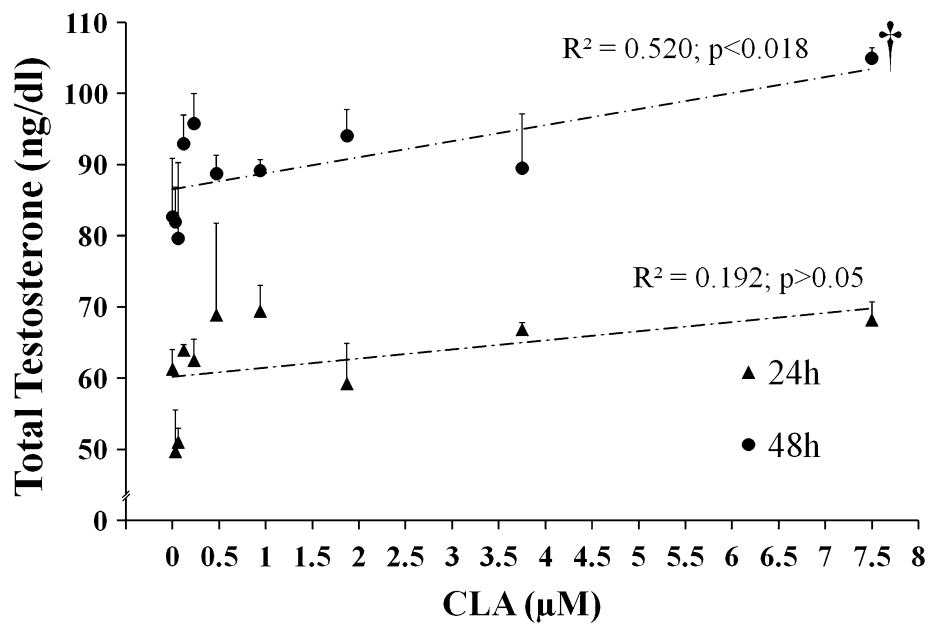

Figure 1. Testosterone production in R2C cells at 24 hours $(\mathbf{\Lambda})$ and 48 hours $(\boldsymbol{\square})$ at different CLA concentrations. All values are means \pm SEM. CLA concentrations used: $0,0.03,0.06,0.12,0.24,0.48,0.95,1.90,3.75$, and $7.5 \mu \mathrm{M}$. The dotted line indicates a linear trend line for testosterone synthesis at 24 and 48 hours. †Significantly different than $0,0.03$, and $0.06 \mu \mathrm{M}$. CLA, conjugated linoleic acid.

\section{Serum Hormones and Sex} Hormone-Binding Globulin

The serum hormones and SHBG concentration results are shown in Figure 2. There was a significant interaction of the resistance exercise bout on total testosterone $(p<0.05)$ and cortisol levels $(p<0.05)$. The total testosterone and cortisol concentration increased significantly at the end of the exercise bouts, although the post hoc analysis did not indicate any significant difference between pre and post resistance exercise bouts. After the resistance exercise bouts, total testosterone increased moderately (ES = moderate), whereas after CLA supplementation, there was a large increase in total testosterone (ES = large). The

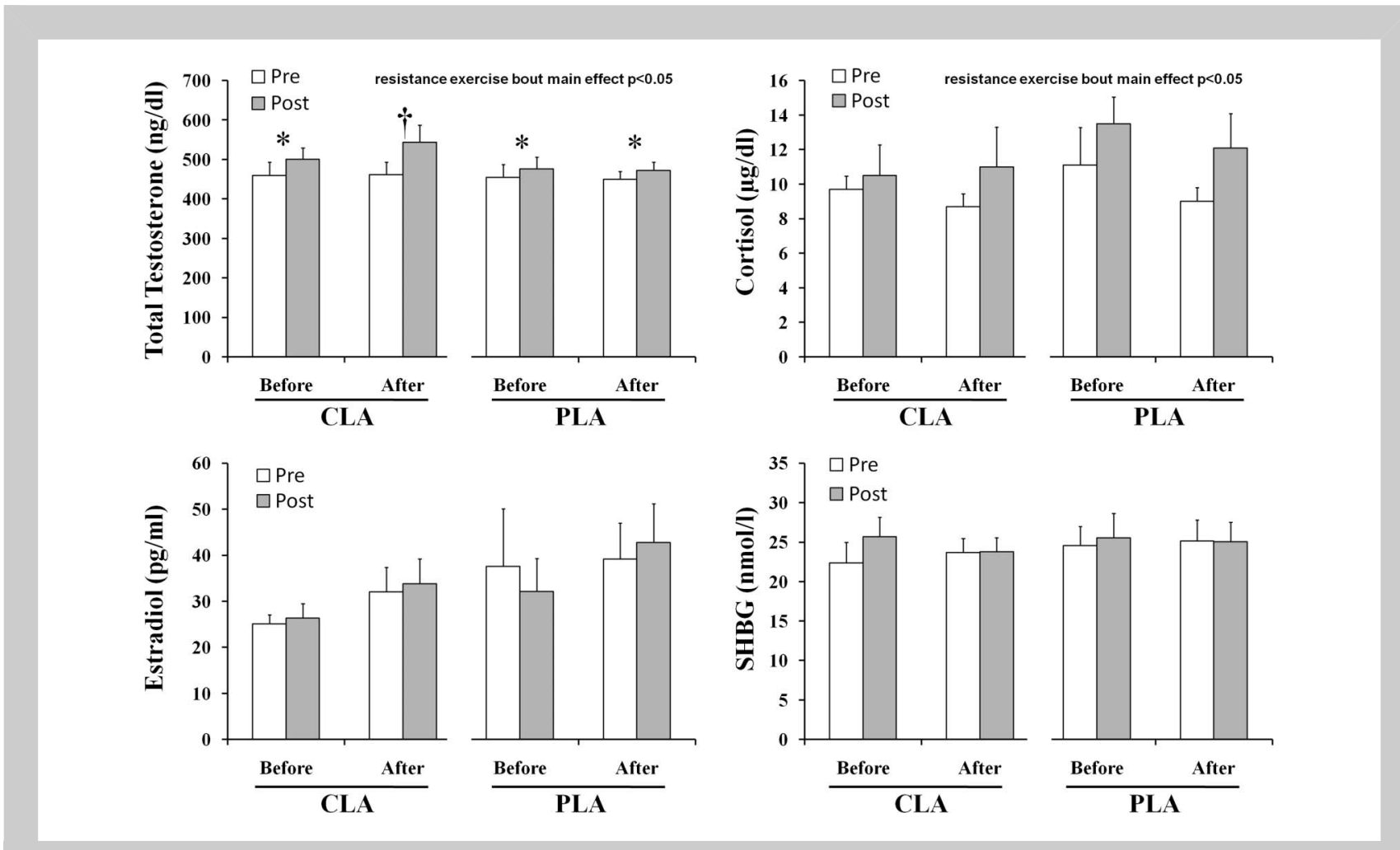

Figure 2. Serum hormones and SHBG. All values are means \pm SEM. *Moderate ES between pre and post. †Large ES. Before, at the beginning of supplementation; after, at the end of supplementation; CLA, conjugated linoleic acid supplementation; ES, effect size; PLA, placebo supplementation; post, post resistance exercise bout; pre, pre resistance exercise bout; SHBG, sex hormone-binding globulin. 
TABLE 2. Training volume.* $\dagger$

\begin{tabular}{lccrr}
\hline & Before PLA & After PLA & Before CLA & After CLA \\
\hline Leg press, $\mathrm{kg}$ & $4,031 \pm 1,233$ & $4,253 \pm 1,128$ & $3,912 \pm 1,179$ & $4,193 \pm 1,547$ \\
Leg curl, kg & $926 \pm 215$ & $1,023 \pm 284$ & $927 \pm 210$ & $942 \pm 239$ \\
Leg extension, kg & $1,203 \pm 317$ & $1,307 \pm 353$ & $1,382 \pm 622$ & $1,373 \pm 675$ \\
Lat pull-down, kg & $2,033 \pm 450$ & $1,988 \pm 478$ & $1,959 \pm 554$ & $2,161 \pm 354$ \\
Bench press, kg & $2,296 \pm 559$ & $2,126 \pm 631$ & $2,222 \pm 557$ & $23,11 \pm 542$ \\
Shoulder press, kg & $1,646 \pm 741$ & $1,830 \pm 787$ & $1,551 \pm 670$ & $1,659 \pm 423$ \\
Barbell bicep curl, kg & $952 \pm 217$ & $1,003 \pm 202$ & $943 \pm 203$ & $951 \pm 209$ \\
Supine triceps extension, kg & $939 \pm 274$ & $1,051 \pm 447$ & $1,015 \pm 420$ & $1,046 \pm 431$ \\
Total volume, kg & $13,637 \pm 3,223$ & $14,222 \pm 3,318$ & $14,306 \pm 3,671$ & $15,170 \pm 3,851$ \\
\hline
\end{tabular}

${ }^{*} \mathrm{CLA}$, conjugated linoleic acid supplementation; PLA, placebo supplementation.

$\dagger$ All values are means $\pm S D$.

large ES in total testosterone level after CLA supplementation was not induced by a fluid shift because there was no difference in plasma and red cell volume among the 4 resistance exercise bouts. The estradiol and SHBG plasma levels did not change in relation to supplementation, training, or resistance exercise bouts.

\section{Training Volume}

No significant differences were observed among the trials in sum or with each exercise lifting volume (Table 2).

\section{Body Composition}

The absolute data on body composition are shown in Figure 3. There were no significant differences in total body mass, body
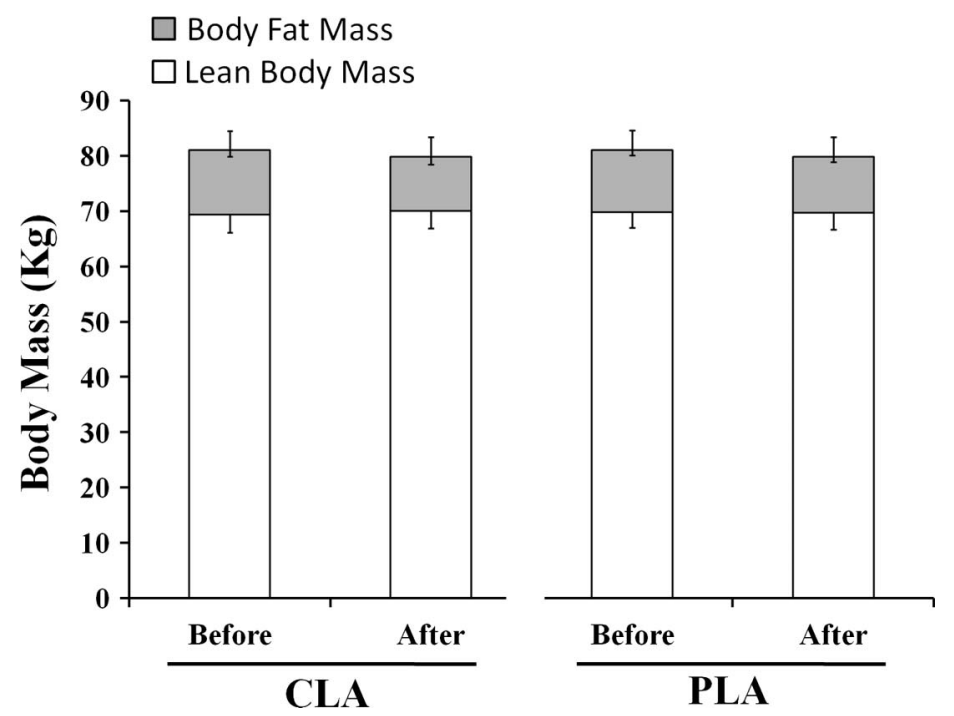

Figure 3. Body composition. All values are means \pm SEM. After, at the end of supplementation; Before, at the beginning of supplementation; CLA, conjugated linoleic acid supplementation; PLA, placebo supplementation. SEs are indicated with error bars, above for total body mass and below for lean body mass and body fat mass. fat mass, and lean body mass before and after the CLA and PLA supplementation. No difference was observed in intraand extracellular water (data not shown).

\section{Discussion}

The possible positive effect of CLA supplementation on the body composition of sedentary or physically active subjects has been previously reported (5); however, our study is the first that provides a set of laboratory data in vitro and in vivo and may yield some insight into the possible mechanisms induced by exercise training and CLA in the modulation of testosterone levels in the blood. Considering the lipolytic effect of the CLA that is mediated by an increase in the level of the perilipin A protein and taking into account the role of perilipins in the pathways leading to steroid hormone synthesis, we investigated the effect of this supplementation on the levels of testosterone in vitro in a cell line derived from Leydig cells and in vivo in the blood of physically active subjects after a bout of resistance exercise. The results of this study will help practitioners and coaches to understand if the CLA supplementation can be useful to increase the testosterone synthesis immediately after an acute session of resistance exercise affecting the anabolic phase followed by each strength training session. It has previously been suggested that the acute elevation of testosterone could mediate an anabolic phase after each strength training session, 
Journal of Strength and Conditioning Research" $\mid$ www.nsca-jscr.org

when the muscle is exposed to the anabolic hormone only for a short period (13).

Conjugated linoleic acid induced an increase in testosterone production in our in vitro experiments. This increase in testosterone production after 48 hours of treatment is proportional to the increase in CLA concentration. The mechanism by which CLA may induce an increase in testosterone level is not known. In normal Leydig cells, testosterone production is dependent on cholesteryl esterase activation by cAMP (22) and lipid hydrolysis depends on dislocation of phosphorylated perilipin $\mathrm{A} / \mathrm{C}$ proteins from the membrane of the lipid droplet (4). The role of perilipin in testosterone synthesis is poorly understood, but it should represent only one of the complex steps of the testosterone synthesis pathway. Considering that the cAMP signaling pathway is inhibited in these cells (22) and also that testosterone is increased after CLA treatment, we can postulate that CLA may induce the activation of perilipin proteins in a cAMP independent manner; therefore, we hypothesize that CLA may have an effect on the steps by which cholesteryl esterase leads to testosterone synthesis in the cytoplasm.

In light of this rise in testosterone synthesis after CLA treatment, we decided to investigate whether CLA supplementation may directly influence testosterone blood levels before and after a resistance exercise bout in humans. The human model was conducted using a resistance exercise bout protocol previously used in literature (3) to increase total testosterone in men. In our study, an overall significant increase in total testosterone and cortisol levels was observed after the resistance exercise, although no difference was seen between interventions. The testosterone and the cortisol results seem to suggest that the resistance exercise program was not markedly stressful for our subjects (i.e., young healthy physically active men). In fact, to induce an acute testosterone response, an acute resistance exercise program needs to contain higher volume and higher metabolic demand in relation of the sex and the age of the subjects tested (23). In humans, the blood level of total testosterone after CLA supplementation after the resistance exercise bouts did not increase significantly as in vitro, although the ES analysis showed a large increase in total testosterone level after CLA supplementation and a moderate increase after placebo supplementation. Conjugated linoleic acid supplementation did not affect the estradiol, cortisol, and SHBG blood levels either before or after a resistance exercise bout.

The data obtained in in vivo do not support our in vitro results but, although there was not a significant increase in testosterone synthesis induced by CLA supplementation, our data showed that after a resistance exercise bout and CLA supplementation, there was a potential slightly higher acute elevation of total testosterone. Furthermore, 2 potential limitations of the in vivo study need to be taken into consideration. The in vitro experiments are indicative of a direct effect of CLA on testosterone synthesis, but we do not know the amount of CLA that accumulates in the testis interstitium and what the active doses on Leydig cells in vivo are. To perform our in vivo experiments, we used doses previously used in literature to test CLA on body mass composition and suggested by the CLA food supplement manufacturers, and it is clear that different doses and dosages need to be tested to understand the effect of CLA on testosterone synthesis in vivo. Another potential limitation in the lack of an effect of CLA on testosterone in vivo could be because of the insufficient exercise stimuli induced by the chosen resistance exercise program in our participants.

Studies conducted in animals have shown that CLA is effective in reducing body fat mass, increasing insulin sensitivity, and decreasing plasma glucose levels (12). These results have led to an increased interest as to whether CLA would have the same effects in humans. The studies that investigate the effect of CLA supplementation in people who are overweight indicate that CLA helps to reduce body fat mass without affecting BMI and body weight (9). The effects of CLA supplementation on the body composition of trained subjects, as reported in the literature, are controversial. Pinkoski et al. (21) observed a relatively small increase of lean body mass and a decrease of body fat mass in 76 moderately physically active subjects fed with CLA for 7 weeks, whereas Kreider et al. (18) showed that 4 weeks of CLA supplementation had no effects on the body composition of 23 bodybuilders. The 3 weeks of CLA supplementation did not induce significant changes in total body weight and body composition in our subject. Our results support the data of Kreider et al. (18), showing that CLA supplementation for a short period (3-4 weeks) combined with exercise does not affect body weight and composition.

In conclusion, our results in vitro demonstrated that CLA supplementation may induce an increase in the testosterone levels, whereas, from the in vivo experiment, it is difficult to generalize that CLA supplementation does not affect testosterone synthesis. As acute hormonal responses show the type and the level of stress affecting the homeostasis of a specific population (17), we can conclude that in young healthy physical active men, CLA supplementation does not significantly increase testosterone synthesis after an unmarkedly stressful bout of acute resistance exercise. Additional research on the effect of CLA on perilipins in the pathway leading testosterone synthesis is important to elucidate the possible mechanism by which CLA can help resistance athletes or people who are overweight.

\section{Practical Applications}

The results of the present study show that CLA may have an effect on testosterone synthesis, although only a potentially slight increase in total testosterone compared with the placebo was observed in physically active men. It means that CLA might be used as an ergogenic aid to increase the anabolic effect of a resistance exercise bout, but further research is needed to understand the physiological relevance of this slight effect on testosterone. 


\section{ACKNOWLEDGMents}

The authors would like to thank the subjects who participated in the study. The authors also thank Dr. N. E. Brooks, Dr. R. Smith, and Mr. A. W. Isaacs (Department of Physiological Science, Stellenbosch University, South Africa) for reading and commenting on our article. This study was funded by MIUR ex 60\% Prof. Farina Felicia and MIUR ex 60\%. This study was funded through a research grant provided to Prof. F. Farina and Dr. G. Morici from Ministero dell'Universita' e della Ricerca Scientifica ex-60\%.

\section{REFERENCES}

1. Bhattacharya, A, Rahman, MM, Sun, D, Lawrence, R, Mejia, W, McCarter, R, O'Shea, M, and Fernandes, G. The combination of dietary conjugated linoleic acid and treadmill exercise lowers gain in body fat mass and enhances lean body mass in high fat-fed male Balb/C mice. J Nutr 135: 1124-1130, 2005.

2. Bird, SP and Tarpenning, KM. Influence of circadian time structure on acute hormonal responses to a single bout of heavy-resistance exercise in weight-trained men. Chronobiol Int 21: 131-146, 2004.

3. Bird, SP, Tarpenning, KM, and Marino, FE. Effects of liquid carbohydrate/essential amino acid ingestion on acute hormonal response during a single bout of resistance exercise in untrained men. Nutrition 22: 367-375, 2006

4. Brasaemle, DL, Subramanian, V, Garcia, A, Marcinkiewicz, A, and Rothenberg, A. Perilipin A and the control of triacylglycerol metabolism. Mol Cell Biochem 326: 15-21, 2009.

5. Campbell, B and Kreide, RB. Conjugated linoleic acids. Curr Sports Med Rep 7: 237-241, 2008.

6. Chung, S, Brown, JM, Sandberg, MB, and McIntosh, M. Trans-10, cis-12 CLA increases adipocyte lipolysis and alters lipid dropletassociated proteins: Role of mTOR and ERK signaling. J Lipid Res 46: 885-895, 2005.

7. Di Felice, V, Macaluso, F, Montalbano, A, Gammazza, AM, Palumbo, D, Angelone, T, Bellafiore, M, and Farina, F. Effects of conjugated linoleic acid and endurance training on peripheral blood and bone marrow of trained mice. J Strength Cond Res 21: 193-198, 2007.

8. Dill, DB, and Costill, DL. Calculation of percentage changes in volumes of blood, plasma, and red cells in dehydration. J Appl Physiol 37: 247-248, 1974.

9. Egras, AM, Hamilton, WR, Lenz, TL, and Monaghan, MS. An evidence-based review of fat modifying supplemental weight loss products. J Obes 2011;2011. pii: 297315.

10. Elmlinger, MW, Kuhnel, W, Wormstall, H, and Doller, PC. Reference intervals for testosterone, androstenedione and SHBG levels in healthy females and males from birth until old age. Clin Lab 51: 625-632, 2005.
11. Faulconnier, Y, Arnal, MA, Patureau Mirand, P, Chardigny, JM, and Chilliard, Y. Isomers of conjugated linoleic acid decrease plasma lipids and stimulate adipose tissue lipogenesis without changing adipose weight in post-prandial adult sedentary or trained Wistar rat. J Nutr Biochem 15: 741-748, 2004.

12. Gaullier, JM, Halse, J, Hoye, K, Kristiansen, K, Fagertun, H, Vik, H, and Gudmundsen, O. Conjugated linoleic acid supplementation for 1 y reduces body fat mass in healthy overweight humans. Am J Clin Nutr 79: 1118-1125, 2004.

13. Hansen, S, Kvorning, T, Kjaer, M, and Sjogaard, G. The effect of short-term strength training on human skeletal muscle: The importance of physiologically elevated hormone levels. Scand J Med Sci Sports 11: 347-354, 2001.

14. Jensky-Squires, NE, Dieli-Conwright, CM, Rossuello, A, Erceg, DN McCauley, S, and Schroeder, ET. Validity and reliability of body composition analysers in children and adults. BrJ Nutr 100: $859-865,2008$

15. Jeukendrup, AE, and Aldred, S. Fat supplementation, health, and endurance performance. Nutrition 20: 678-688, 2004.

16. Kelly, GS. Conjugated linoleic acid: A review. Altern Med Rev 6: 367-382, 2001

17. Kraemer, WJ, Häkkinen, K, Newton, RU, Nindl, BC, Volek, JS, McCormick, M, Gotshalk, LA, Gordon, SE, Fleck, SJ, Campbell, WW, Putukian, M, and Evans, WJ. Effects of heavy-resistance training on hormonal response patterns in younger vs. older men. J Appl Physiol 87: 982-992, 1999.

18. Kreider, RB, Ferreira, MP, Greenwood, M, Wilson, M, and Almada, AL. Effects of conjugated linoleic acid supplementation during resistance training on body composition, bone density, strength, and selected hematological markers. J Strength Cond Res 16: 325-334, 2002.

19. Mizunoya, W, Haramizu, S, Shibakusa, T, Okabe, Y, and Fushiki, T. Dietary conjugated linoleic acid increases endurance capacity and fat oxidation in mice during exercise. Lipids 40: 265-271, 2005.

20. Nomura, S, Kawanami, H, Ueda, H, Kizaki, T, Ohno, H, and Izawa, T. Possible mechanisms by which adipocyte lipolysis is enhanced in exercise-trained rats. Biochem Biophys Res Commun 295: 236-242, 2002.

21. Pinkoski, C, Chilibeck, PD, Candow, DG, Esliger, D, Ewaschuk, JB, Facci, M, Farthing, JP, and Zello, GA. The effects of conjugated linoleic acid supplementation during resistance training. Med Sci Sports Exerc 38: 339-348, 2006.

22. Servetnick, DA, Brasaemle, DL, Gruia-Gray, J, Kimmel, AR, Wolff, J, and Londos, C. Perilipins are associated with cholesteryl ester droplets in steroidogenic adrenal cortical and Leydig cells. J Biol Chem 270: 16970-16973, 1995.

23. Vingren, JL, Kraemer, WJ, Ratamess, NA, Anderson, JM, Volek, JS and Maresh, CM. Testosterone physiology in resistance exercise and training: The up-stream regulatory elements. Sports Med 40: $1037-1053,2010$. 